\title{
THE TRANSITION FROM DISK TO HALO AS SEEN FROM CORRELATIONS BETWEEN KINEMATICS
}

\author{
AND METALLICITY
}

\author{
S. BARTAS̆IŪTÉ \\ Vilnius University Observatory \\ Ciurlionio 29, Vilnius 2009, Lithuania
}

An investigation has been made to determine the relationship between kinematic and chemical properties of stars covering nearly all population types in the Galaxy. For this purpose, a sample has been taken of about 1150 high-galactic-latitude F-K stars down to $V=14.0$, for which photoelectric seven-color data in the Vilnius medium-band system were collected at the Maidanak Observatory in Uzbekistan, Central Asia. Combining the obtained photometric material with the proper motion and radial velocity data enabled us to compute kinematic parameters of the sample stars and then to make plots of the galactic orbital eccentricities, angular momenta, and velocity dispersions versus $[\mathrm{Fe} / \mathrm{H}]$, with much attention drawn to the region where the transition between the thin disk and halo occurs.

It is found that the kinematic parameters do not appear to vary continuously with abundance over most of the $[\mathrm{Fe} / \mathrm{H}]$ range. In particular, there is an abrupt transition from the thin disk to the thick disk population and from the thick disk to the halo, as most clearly evidenced by the $\sigma_{W},[\mathrm{Fe} / \mathrm{H}]$ trend. Within the thick disk itself, however, stars are found to display a progressive increase of their kinematic parameters with decreasing metallicity, as well as a statistically significant metallicity gradient given formally by $d[\mathrm{Fe} / \mathrm{H}] / d\left|Z_{\max }\right|=-0.21 \pm 0.09 \mathrm{kpc}^{-1}$.

An attempt has also been made to estimate the mean kinematic parameters within the thick disk component, provided the metal abundance range $-1.0 \leq[\mathrm{Fe} / \mathrm{H}]<-0.4$ represents reasonably this population. For the sample of 114 stars that satisfy this metallicity criterion, we have the velocity dispersion tensor $\left(\sigma_{U}, \sigma_{V}, \sigma_{W}\right)=(64 \pm 5,49 \pm 3,42 \pm 3) \mathrm{km} \mathrm{s}^{-1}$ and the mean rotation velocity $181 \pm 5 \mathrm{~km} \mathrm{~s}^{-1}$ (adopting $220 \mathrm{~km} \mathrm{~s}^{-1}$ for the Sun relative to the external frame). 